Article

\title{
Response Surface Optimized Extraction of 1-Deoxynojirimycin from Mulberry Leaves (Morus alba L.) and Preparative Separation with Resins
}

\section{Teng Wang ${ }^{1}$, Cui-Qing $\mathrm{Li}^{2}{ }^{2, *}$, Hong Zhang ${ }^{2}$ and $\mathrm{Ji}-$ Wen $\mathrm{Li}^{3}$}

1 Department of Pharmaceutical Engineering, Beijing Institute of Petro-chemical Technology, Beijing 102617, China

2

Department of Chemical Engineering, Beijing Institute of Petro-chemical Technology, Beijing 102617, China

3 College of Science, Agricultural University of Hebei, Baoding 071001, China

* Author to whom correspondence should be addressed; E-Mail: licuiqing@bipt.edu.cn; Tel./Fax: +86-10-8129-2124.

Received: 26 March 2014; in revised form: 21 May 2014 / Accepted: 21 May 2014 /

Published: 30 May 2014

\begin{abstract}
In the present study, the extraction technology and preparative separation of 1-deoxynojirimycin from mulberry leaves were systematically investigated. Four extraction parameters (ethanol concentration, extraction temperature, extraction time and ratio of solvent to sample) were explored by response surface methodology (RSM). The results indicated that the maximal yield of 1-deoxynojirimycin was achieved with an ethanol concentration of $55 \%$, extraction temperature of $80{ }^{\circ} \mathrm{C}$, extraction time of $1.2 \mathrm{~h}$ and ratio of solvent to sample of 12:1. The extraction yield under these optimum conditions was found to be $256 \mathrm{mg} / 100 \mathrm{~g}$ dry mulberry leaves. A column packed with a selected resin was used to perform dynamic adsorption and desorption tests to optimize the separation process. The results show that the preparative separation of 1-deoxynojirimycin from mulberry leaves can be easily and effectively done by adopting 732 resin. In conclusion, 732 resin is the most appropriate for the separation of 1-deoxynojirimycin from other components in mulberry leaves extracts, and its adsorption behavior can be described with Langmuir isotherms and a two-step adsorption kinetics model. The recovery and purity of 1-deoxynojirimycin in the final product were $90.51 \%$ and $15.3 \%$, respectively.
\end{abstract}

Keywords: 1-deoxynojirimycin; extraction; response surface methodology; resin 


\section{Introduction}

Mulberry leaves (Morus alba L.), cultivated mainly in Asian countries, have drawn the interest of many researchers because they were found to have antihyperglycemic, antioxidant and antimicrobial activities [1-3]. Mulberry leaves are rich in flavonoid, alkaloid and polysaccharide components which are known to be the major bioactive compounds from chemical constituent investigations. Among those, 1-deoxynojirimycin (1-DNJ), which is known as a potent $\alpha$-glycosidase inhibitor, has gained considerable attention for use as a functional or medical additive to control blood glucose [4,5]. These pharmaceutical discoveries led to interest in the exploration of effective extraction and preparative separations of 1-DNJ from mulberry leaves.

In order to prepare bioactive compounds, solvent extraction was often used and the extraction yield is significantly influenced by many factors, such as solvent concentration, extraction temperature, extraction time, and ratio of solvent to sample, etc. [6-8]. The traditional optimization method was single-factor experiments, in which only one factor is varied at a time while others are fixed at constant values. However, this method is time-consuming and cannot evaluate the interaction effects among the various factors. Response surface methodology (RSM) is an effective technique to overcome these problems [9]. It can explore the relationships between the response values and the independent variables and optimize the processes where multiple variables may influence the outputs [10]. Up to now, RSM has been successfully used to model and optimize the biochemical and biotechnological processes related to food systems [11-13].

The future use of 1-DNJ is bright, but the amount of the key constituent in mulberry leaves is as low as only $0.1 \%[14,15]$, hence, a more efficient and simple purification method for 1 -DNJ is required. The purification method with resin is a new promising technology, and has been successfully applied in the preparative separation of natural products [16]. Similarly, such a method is extremely useful for the extraction and separation of the 1-DNJ from mulberry leaves.

The objective of the present study was to investigate the influence of ethanol concentration, extraction temperature, extraction time and ratio of solvent to sample on the yield of 1-DNJ using RSM. Furthermore, the adsorption and desorption properties of 1-DNJ from mulberry leaves with different resins were investigated and an efficient method for the preparative separation of 1-DNJ from mulberry leaves with 732 resin has been developed.

\section{Results and Discussion}

\subsection{Optimization of Extraction Parameters of 1-DNJ}

\subsubsection{Fitting the Response Surface Model}

The effect of four independent variables (ethanol concentration $\left(X_{1}\right)$, extraction temperature $\left(X_{2}\right)$, extraction time $\left(X_{3}\right)$ and ratio of solvent to sample $\left.\left(X_{4}\right)\right)$ on the yield of extraction $(Y)$ was investigated using a four-factor and three levels Box-Behnken design (Table 1). 
Table 1. Experimental design using Box-Behnken and the extraction yields of 1-DNJ.

\begin{tabular}{|c|c|c|c|c|c|}
\hline Number & $X_{1}$ & $X_{2}$ & $X_{3}$ & $X_{4}$ & $Y(\mathrm{mg} / 100 \mathrm{~g}$ dry powder $)$ \\
\hline 1 & 0 & 0 & -1 & -1 & 224 \\
\hline 2 & 0 & 0 & -1 & 1 & 240.8 \\
\hline 3 & 0 & -1 & 0 & -1 & 174.8 \\
\hline 4 & 0 & 0 & 1 & -1 & 248.8 \\
\hline 5 & 0 & 1 & 1 & 0 & 160.4 \\
\hline 6 & 1 & 1 & 0 & 0 & 125.2 \\
\hline 7 & -1 & -1 & 0 & 0 & 105.2 \\
\hline 8 & -1 & 1 & 0 & 0 & 154 \\
\hline 9 & 0 & 0 & 0 & 0 & 249.2 \\
\hline 10 & 1 & 0 & 0 & 1 & 110.8 \\
\hline 11 & 1 & 0 & 1 & 0 & 175.2 \\
\hline 12 & 0 & 0 & 0 & 0 & 244.4 \\
\hline 13 & 0 & 0 & 0 & 0 & 246 \\
\hline 14 & -1 & 0 & 0 & 1 & 250.8 \\
\hline 15 & 1 & 0 & 0 & -1 & 149.2 \\
\hline 16 & 0 & 1 & 0 & -1 & 128.8 \\
\hline 17 & 0 & 1 & -1 & 0 & 127.2 \\
\hline 18 & -1 & 0 & 0 & -1 & 114.8 \\
\hline 19 & 1 & -1 & 0 & 0 & 168.8 \\
\hline 20 & 0 & 0 & 0 & 0 & 245.6 \\
\hline 21 & -1 & 0 & -1 & 0 & 172.8 \\
\hline 22 & 0 & 1 & 0 & 1 & 164.4 \\
\hline 23 & 1 & 0 & -1 & 0 & 114.4 \\
\hline 24 & 0 & -1 & -1 & 0 & 212.8 \\
\hline 25 & 0 & 0 & 1 & 1 & 246.4 \\
\hline 26 & -1 & 0 & 1 & 0 & 104.4 \\
\hline 27 & 0 & -1 & 1 & 0 & 158.8 \\
\hline 28 & 0 & -1 & 0 & 1 & 148.8 \\
\hline 29 & 0 & 0 & 0 & 0 & 249.2 \\
\hline
\end{tabular}

Multiple linear regression analysis was performed based on the results in Table 1 using the following second-order polynomial Equation (1):

$$
Y=\gamma_{0}+\sum_{i=1}^{3} a_{i} X_{i}+\sum_{i=1}^{3} a_{i i} X_{i}^{2}+\sum_{i=1}^{2} \sum_{j=i+1}^{3} a_{i j} X_{i} X_{j}
$$

where $Y$ is the predicted response, $\gamma_{0}$ is a constant, $a_{i}, a_{i j}$ and $a_{i j}$ are respectively the linear, quadratic and interactive coefficients of the model. Accordingly, $X_{i}$ and $X_{j}$ represent the levels of the independent variables, respectively. The response variable and the independent variables are related by the following second-order polynomial equation [Equation (2)]:

$$
\begin{gathered}
Y=246.88-4.86 X_{1}-9.1 X_{2}+0.17 X_{3}+10.13 X_{4}-23.11 X_{1} X_{2}+32.3 X_{1} X_{3}-43.6 \mathrm{X}_{1} \mathrm{X}_{4}+ \\
21.8 X_{2} X_{3}+15.4 X_{2} X_{4}-4.8 X_{3} X_{4}-71.14 X_{1}^{2}-60.69 X_{2}^{2}-16.09 X_{3}^{2}-14.04 X_{4}^{2}
\end{gathered}
$$


The statistics test of the model was performed by "analysis of variance" (ANOVA) and the results are shown in Table 2. The value of the determination coefficient $\left(R^{2}\right)$ of the regression model was 0.8432 , which indicated that $84.32 \%$ of the variations could be explained by the fitted model, suggesting that a closely correlation was achieved as the $R^{2}$ value was higher than 0.8 [17]. The "Adequate Precision" measures the signal to noise ratio and a ratio greater than 4 is desirable. In this study, the ratio was found to be 7.835 , indicating that this model can be used to navigate the design space [18].

The significance of the regression model was checked using the $P$-value and the model would be more significant with a smaller $P$-value. The ANOVA of the quadratic regression model demonstrated that the model was highly significant, as was evident from a very low probability value $(p=0.001)$. Furthermore, the significance of the model was also determined by "Lack of Fit" test, the $F$-value of the "Lack of Fit" was 259.88, which suggested that it was not significant and only a $0.01 \%$ chance could occur due to noise [19].

Additionally, the $p$-value was also used to evaluate the significance of each coefficient, which might indicate the pattern of the interactions between the variables. In this case, the cross product coefficients $\left(X_{1} X_{3}, X_{1} X_{4}\right)$ and the quadratic term coefficients $\left(X_{1}^{2}, X_{2}^{2}\right)$ were significant with a very small $p$-value $(p<0.05)$, while the other term coefficients were not significant $(p>0.05)$.

Table 2. The regression coefficients and ANOVA results.

\begin{tabular}{|c|c|c|c|c|c|}
\hline Source & DF & Sum of Square & Mean Square & $F$-value & $p$-value \\
\hline Model & 14 & $67,638.64$ & 4831.33 & 5.3781 & 0.0017 \\
\hline$X_{1}$ & 1 & 284.21 & 284.21 & 0.3163 & 0.5827 \\
\hline$X_{2}$ & 1 & 993.72 & 993.72 & 1.1061 & 0.3107 \\
\hline$X_{3}$ & 1 & 0.33 & 0.333 & 0.0004 & 0.9849 \\
\hline$X_{4}$ & 1 & 1232.21 & 1232.21 & 1.3716 & 0.2611 \\
\hline$X_{1} X_{2}$ & 1 & 2134.44 & 2134.44 & 2.3760 & 0.1455 \\
\hline$X_{1} X_{3}$ & 1 & 4173.16 & 4173.16 & 4.6455 & 0.0490 \\
\hline$X_{1} X_{4}$ & 1 & 7603.84 & 7603.84 & 8.4645 & 0.0114 \\
\hline$X_{2} X_{3}$ & 1 & 1900.96 & 1900.96 & 2.1161 & 0.1678 \\
\hline$X_{2} X_{4}$ & 1 & 948.64 & 948.64 & 1.0560 & 0.3216 \\
\hline$X_{3} X_{4}$ & 1 & 92.16 & 92.16 & 0.1026 & 0.7535 \\
\hline$X_{1}^{2}$ & 1 & $32,827.46$ & $32,827.45$ & 36.5431 & $<0.0001$ \\
\hline$X_{2}^{2}$ & 1 & $23,891.52$ & $23,891.5$ & 26.5957 & 0.0001 \\
\hline$X_{3}^{2}$ & 1 & 1679.27 & 1679.27 & 1.8693 & 0.1931 \\
\hline$X_{4}^{2}$ & 1 & 1278.62 & 1278.62 & 1.4233 & 0.2527 \\
\hline Lack of Fit & & - & - & 259.88 & 0.0001 \\
\hline Pure Error & 4 & 19.28 & 4.83 & & \\
\hline Total & 28 & $80,215.13$ & & & \\
\hline
\end{tabular}

\subsubsection{Analysis of the Response Surface}

$3 \mathrm{D}$ response surface plots provide a method to visualize the relationship between responses and experimental levels of each variable and the type of interactions between two test variables. Different 
shapes of the contour plots indicated different interactions between the variables. Circular contour plot indicated that the interactions between the corresponding variables were negligible, while elliptical contour plot indicated that the interactions between the corresponding variables were significant [20,21]. As shown in Figure 1a, when extraction time $\left(X_{3}\right)$ and ratio of solvent to sample $\left(X_{4}\right)$ were kept at zero level, ethanol concentration $\left(X_{1}\right)$ and extraction temperature $\left(X_{2}\right)$ were showed reciprocal interaction on the extraction yield. When extraction temperature $\left(X_{2}\right)$ kept at lower level, the yield of 1-DNJ increased at first, and then decreased with the increase of ethanol concentration $\left(X_{1}\right)$. The circular contour plot indicated that the interactions between ethanol concentration $\left(X_{1}\right)$ and extraction temperature $\left(X_{2}\right)$ were insignificant. Likewise, the interaction of the extraction time $\left(X_{3}\right)$ and ratio of solvent to sample $\left(X_{4}\right)$ were demonstrated insignificantly on the extraction yield of 1-DNJ from Figure 1f. Figure $1 \mathrm{~b}$ shows the influence of ethanol concentration $\left(X_{1}\right)$ and extraction time $\left(X_{3}\right)$ demonstrating quadratic effects on the yield of 1-DNJ when the other two variables were fixed at zero level. The mutual interaction between ethanol concentration $\left(X_{1}\right)$ and extraction time $\left(X_{3}\right)$ was significant which may be concluded by the type of the contour plot.

Figure 1c shows the response surface at varying ethanol concentration $\left(X_{1}\right)$ and ratio of solvent to sample $\left(X_{4}\right)$ with the fixed extraction temperature (zero level) and extraction time (zero level). It can be concluded that the extraction yield of 1-DNJ was affected significantly by ethanol concentration $\left(X_{1}\right)$ and ratio of solvent to sample $\left(X_{4}\right)$. The extraction yield increased with the increase of ratio of solvent to sample $\left(X_{4}\right)$ when ethanol concentration $\left(X_{1}\right)$ was kept at lower level, while decreased with the increase of ratio of solvent to sample $\left(X_{4}\right)$ when ethanol concentration $\left(X_{1}\right)$ was kept at higher level.

Figure $1 \mathrm{~d}$ shows the interactive influences of the extraction temperature $\left(X_{2}\right)$ and extraction time $\left(X_{3}\right)$ while the other two variables were kept at zero level. The yield of 1-DNJ was slightly decreased with the increase of extraction time $\left(X_{3}\right)$ when extraction temperature $\left(X_{2}\right)$ was kept the lower level. Furthermore, it can be concluded that the interactive influence between extraction temperature $\left(X_{2}\right)$ and extraction time $\left(X_{3}\right)$ was insignificant. Likewise, Figure 1e showed that the interaction of extraction temperature $\left(X_{2}\right)$ and ratio of solvent to sample $\left(X_{4}\right)$ were demonstrated insignificantly on the extraction yield of 1-DNJ. The extraction yield of 1-DNJ increased slightly with the increase of ratio of solvent to sample $\left(X_{4}\right)$ when extraction temperature $\left(X_{2}\right)$ was kept at lower level.

\subsubsection{Validation of the Model Equation}

The suitability of the model for predicting the optimum response values was tested by using the optimal conditions with small modifications. The maximum predicted yield and experimental yield of 1-DNJ were presented in Table 3. The experiments by using the predicted optimum extraction conditions for 1-DNJ were as follows: ethanol concentration of $55.2 \%$, extraction temperature of $80.4{ }^{\circ} \mathrm{C}$, extraction time of $1.2 \mathrm{~h}$, ratio of solvent to sample of $12: 1$, and the model predicted a maximum yield of $256 \mathrm{mg} / 100 \mathrm{~g}$ dry powder. In order to ensure the predicted result, validation experiment was performed by using the modified conditions: the concentration of ethanol of $55 \%$, extraction temperature of $80{ }^{\circ} \mathrm{C}$, extraction time of $1.2 \mathrm{~h}$, ratio of solvent to sample of $12: 1$. A mean value of $255 \pm 2.54 \mathrm{mg} / 100 \mathrm{~g}$ dry powder was obtained from validation experiments. The good correlation among these results confirmed that the response model was adequate for reflecting the expected optimization. The results 
of analysis indicated that the experimental values were in agreement with the predicted one, and also suggested that the model was satisfactory and accurate.

Figure 1. Response surface plots showing the effect of ethanol concentration $\left(X_{1}\right)$, extraction temperature $\left(X_{2}\right)$, extraction time $\left(X_{3}\right)$ and ratio of solvent to sample $\left(X_{4}\right)$ on the yield of 1-DNJ.
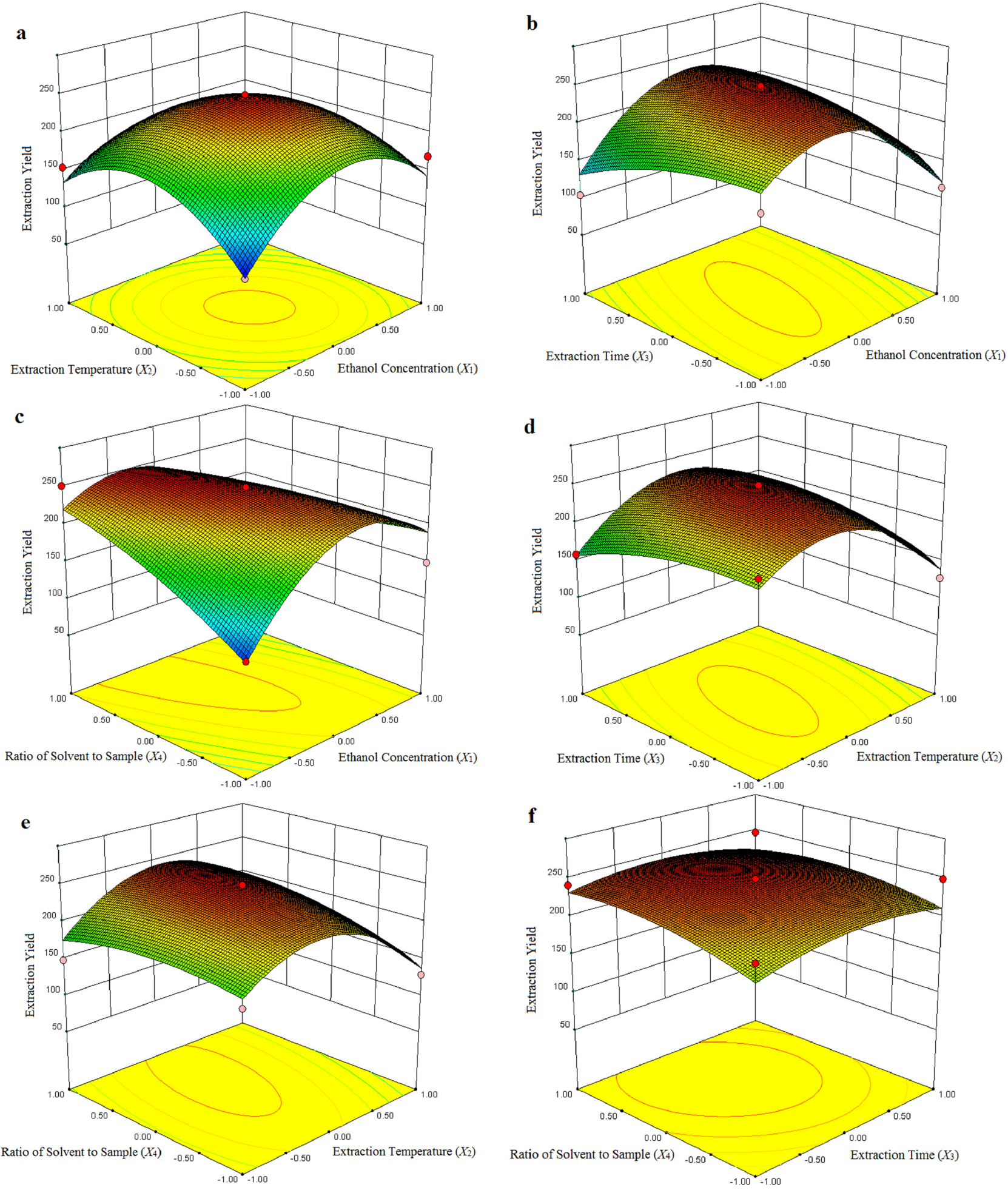
Table 3. Predicted and experimental values of the response under the optimal extraction conditions.

\begin{tabular}{cccccc}
\hline & \multicolumn{2}{c}{ Extraction variables } & & $\begin{array}{c}\text { Predicted yield } \\
(\mathbf{m g} / \mathbf{1 0 0} \text { g dry powder) }\end{array}$ & $\begin{array}{c}\text { Experimental yield } \\
(\mathbf{m g} / \mathbf{1 0 0} \text { g dry powder) }\end{array}$ \\
\cline { 1 - 4 }$X_{1}(\%)$ & $X_{2}\left({ }^{\circ} \mathrm{C}\right)$ & $X_{3}(\mathrm{~h})$ & $X_{4}(\mathrm{~g} / \mathrm{mL})$ & 256 & $255 \pm 2.54^{\mathrm{a}}$ \\
\hline 55 & 80 & 1.2 & 12 & & \\
\hline \multicolumn{5}{c}{${ }^{\mathrm{a}}$ Mean \pm standard deviation $(\mathrm{n}=5)}$.
\end{tabular}

\subsection{Static Adsorption Capacity, Adsorption Ratio, Desorption Ratio and Recovery}

The selectivity of resins was based on the capacities of adsorption and desorption, and radio of desorption. The adsorption capacity, adsorption ratio, desorption ratio and recovery of 1-DNJ with different resins were shown in Figure 2. It can be seen that the adsorption capacities of 732, 201 and AB-8 resins were obviously higher than those of other resins. However, the desorption ratio and recovery of 201 resin was the lowest in all resins. While the desorption ratio and recovery of 152 resin was fairly prominent, the adsorption capacity was barely satisfactory. Therefore, 732 and AB-8 resins were selected for the further investigation of adsorption behavior of 1-DNJ from mulberry leaves.

Figure 2. Adsorption capacity, adsorption ratio, desorption ratio and recovery of 1-DNJ with different resins.
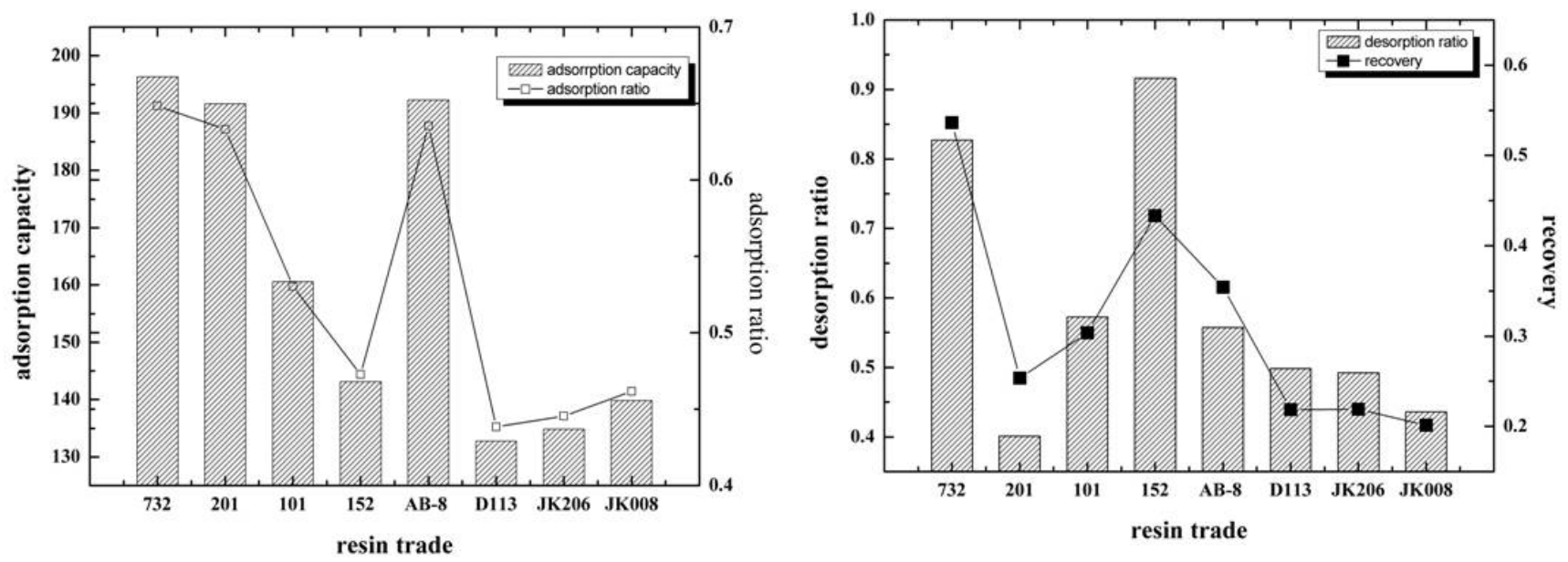

\subsection{Static Adsorption Isotherms of 1-DNJ with 732 and AB-8 Resins}

The most suitable initial concentration was achieved by plot between the adsorption ration and the initial concentration of 1-DNJ as shown in Figure 3. It can be seen that the adsorption capacity of 732 and $\mathrm{AB}-8$ resins increased with the initial concentration of 1-DNJ and reached the saturation plateau when the initial concentrations of 1-DNJ were 9.84 and $8.68 \mathrm{mg} / \mathrm{mL}$.

In research on adsorption processes, the Langmuir, Freundlich and Toth isotherms are often used to illustrate the adsorption behavior of solutes in the separation. Adsorption isotherms of 732 and AB-8 resins were conducted at the temperatures of 20,30 and $40{ }^{\circ} \mathrm{C}$.

The isotherms and parameters were obtained from the Langmuir, Freundlich and Toth equations as shown in Figure 4. It can be seen that the correction coefficients of then Langmuir, Freundlich and Toth equations with 732 resin were obviously higher than AB-8 resin. In the comprehensive consideration 
of the adsorption capacity and desorption ration, 732 resin was selected for the separation of 1-DNJ from mulberry leaves.

Figure 3. The relationship between the adsorption ratio and the initial concentration of 1-DNJ.

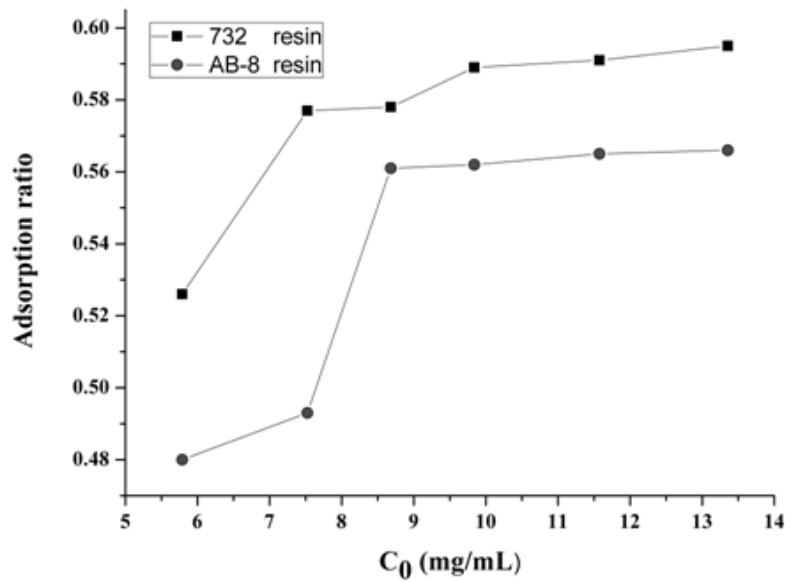

Figure 4. The adsorption isotherms of 1-DNJ with 732 and AB-8 resins at different temperatures.
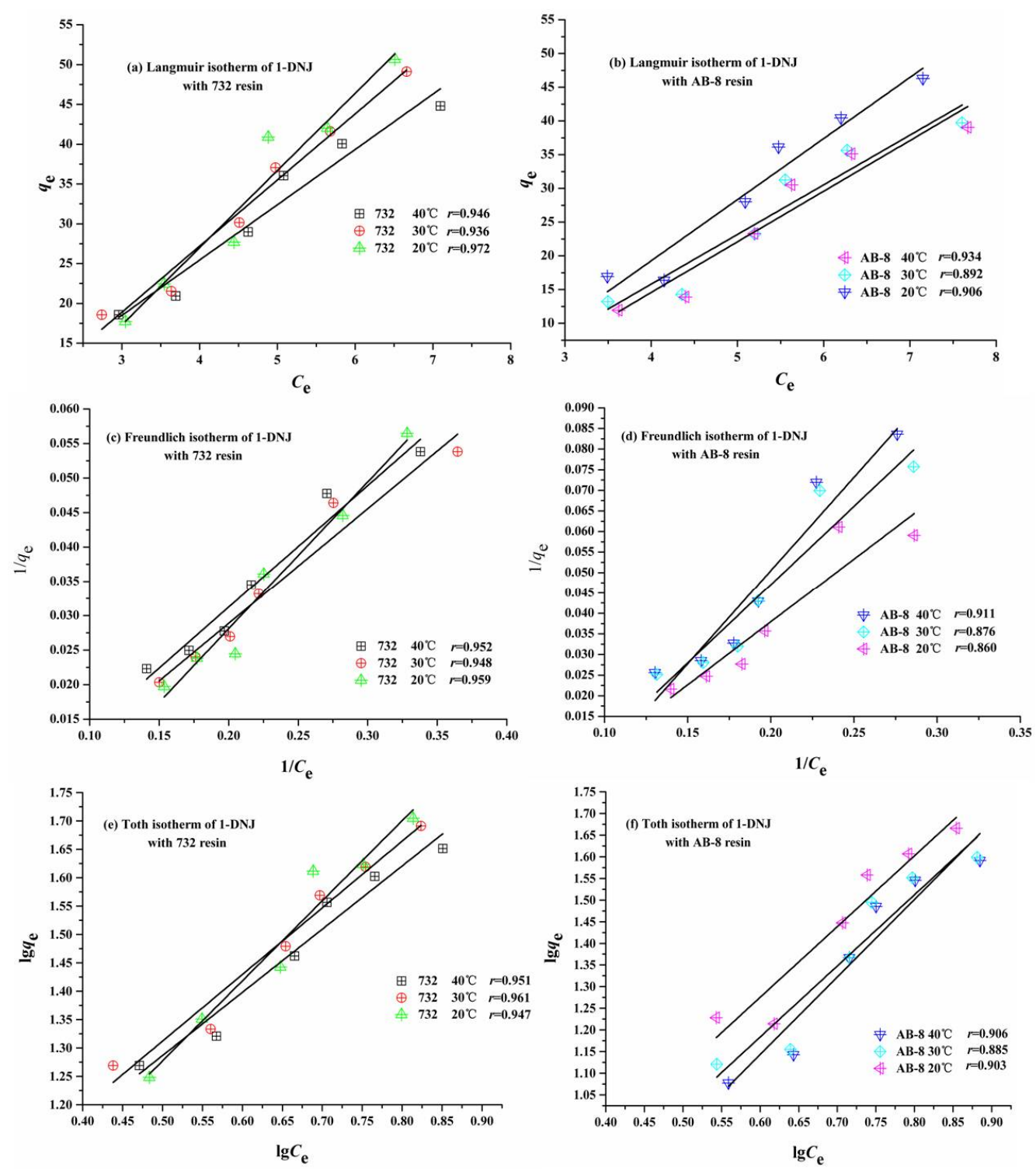
Furthermore, the equilibrium adsorption isotherms with 732 resin were highly fitted to the Langmuir models. The Langmuir model assumes monomolecular layer adsorption with the homogeneous distribution of adsorption energies while there is no mutual interaction between adsorbed molecules. The Freundlich model can be used to describe the adsorption behavior of monomolecular layer as well as that of the multimolecular layer [22]. All the adsorption sites on the surface of resin were equivalent and independent, the adsorbates rapidly cover the surface of 732 resin and form one monolayer, the rate during the adsorption process depends on the concentration of the adsorbate in the solution.

\subsection{Static Adsorption Kinetic of 1-DNJ with 732 Resin}

In order to reveal the adsorption behavior of 1-DNJ with 732 resin further, the kinetic curve was calculated as shown in Figure 5. It can be seen that the adsorption capacity of 732 resin increased with the increasing adsorption time. In the first two hours, the adsorption capacity increased rapidly and reached the equilibrium at about $4 \mathrm{~h}$.

Figure 5. Adsorption kinetic curve of 1-DNJ in mulberry leaves with 732 resin.

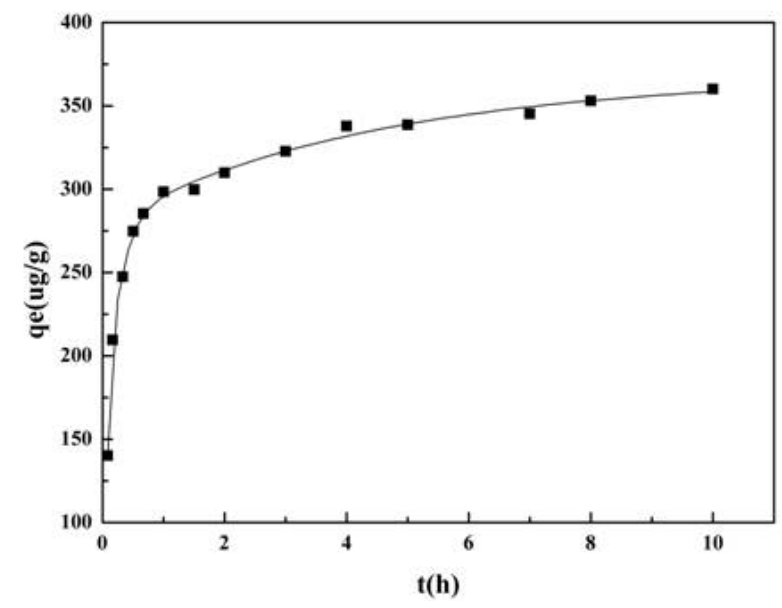

The adsorption process can be seen as the redistribution of the solute molecules between the adsorbents and the liquid phase. Figure 6 shows the adsorption kinetic model which was simulated by the experimental data of the adsorption of 1-DNJ with 732 resin. As can be seen from Figure 6, the adsorption process can be considered as a two-step process. For the two steps, the adsorption rate constants $K_{1}>K_{2}$, which demonstrates the adsorption process of 1-DNJ with 732 resin may be explained by "an initial fast step followed by a slow step". In the process of adsorption, the surface coverage by the adsorbed 1-DNJ molecules increases rapidly due to the formation of the first adlayer at the early stage and then trends to gentle by the formation of the second adlayer at the later stage [23].

\subsection{Dynamic Adsorption Curve of 1-DNI with 732 Resin}

The flow rate of the sample solution is one of the factors influencing the dynamic adsorption. The dynamic adsorption curves of 1-DNJ with 732 resin under different flow rates were obtained based on the linear relationship between $\ln \left[C /\left(C_{0}-C\right)\right]$ and $\mathrm{t}$, as shown in Figure 7. As can be seen from Figure 7, the dynamic leakage time and the adsorption capacity increased as the flow rate increased. In the comprehensive consideration of the dynamic leakage time, adsorption capacity and 
regression coefficient of adsorption curve, the flow rate of $5 \mathrm{~mL} / \mathrm{min}$ was selected for the dynamic adsorption of 1-DNJ with 732 resin.

Figure 6. Adsorption rate constants in two-step adsorption kinetic model with 732 resin.

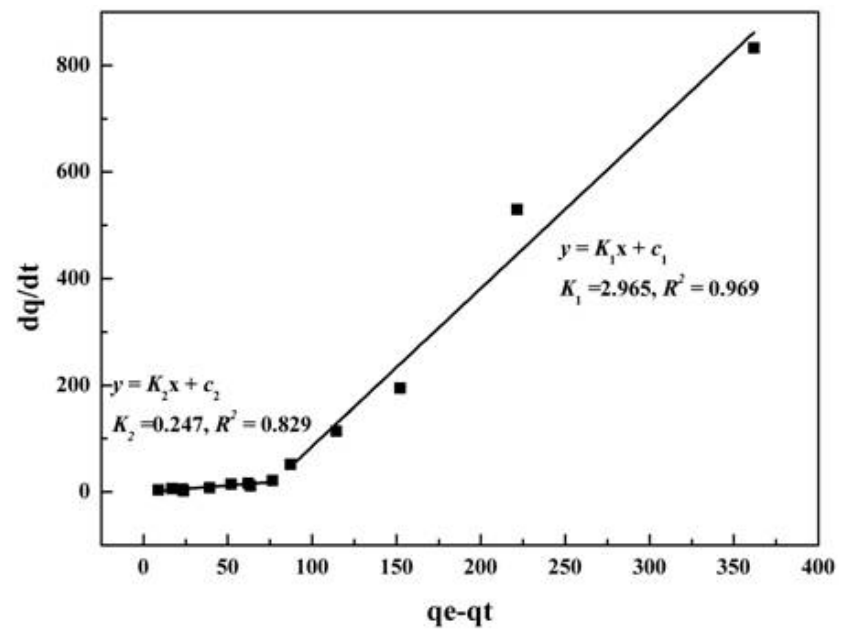

Figure 7. Dynamic adsorption curves of 1-DNJ with 732 resins under different flow rates.

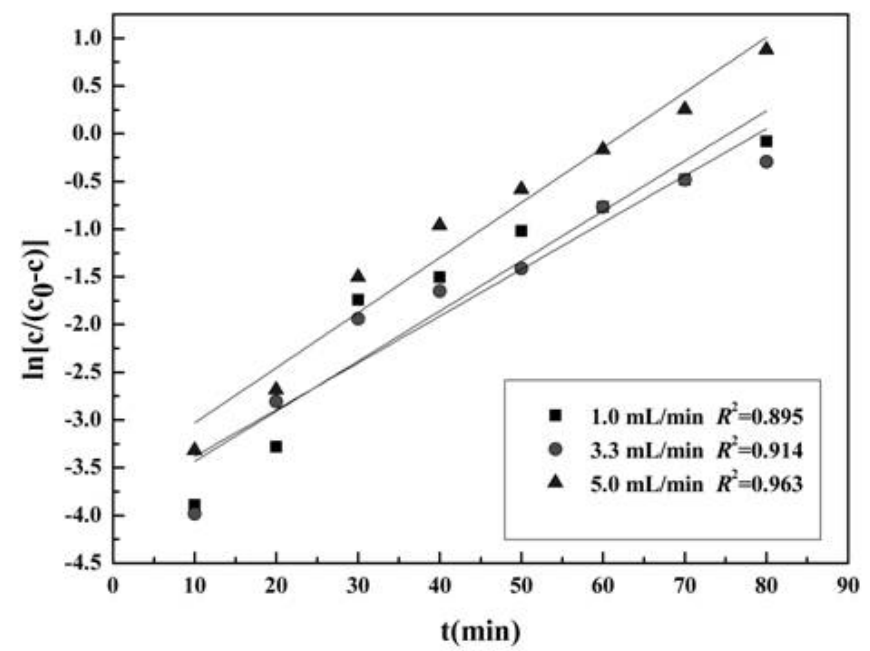

\subsection{Dynamic Desorption Curve of 1-DNJ with 732 Resin}

The dynamic desorption curves were obtained by plotting the amount of 1-DNJ desorbed and the desorption time. As can be seen from Figure 8, the flow rate is one of the important factors influencing the dynamic desorption curves, and the recovery of 1-DNJ is expected to decrease with increasing flow rate. Meanwhile, it shows that the dynamic desorption curves fitted to the Pearson IV equation as shown in Figure 8. In the comprehensive consideration of the desorption time, recovery and purity of 1-DNJ, the flow rate of desorption solution of $1.0 \mathrm{~mL} / \mathrm{min}$ was selected as the most suitable flow rate for the dynamic desorption process. 
Figure 8. Dynamic desorption curves of 1-DNJ with 732 resins under different flow rates.

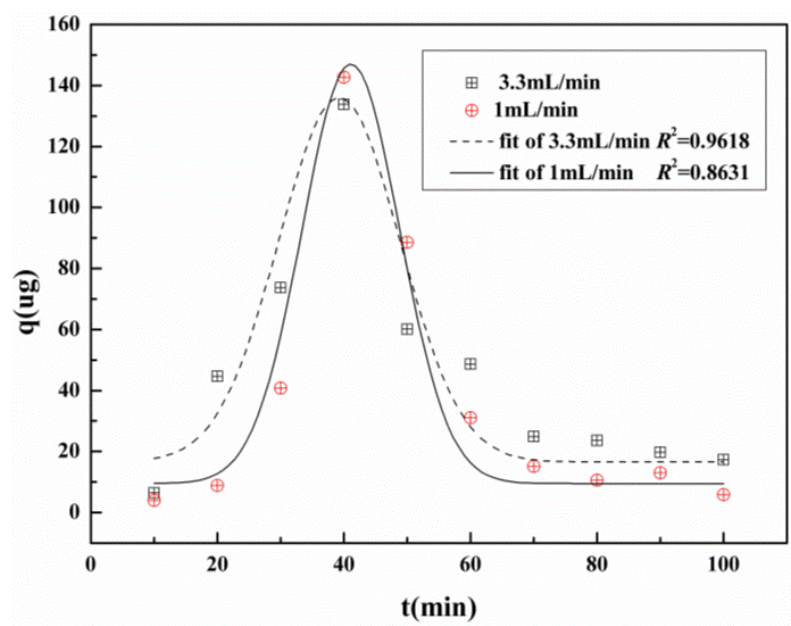

\subsection{The Results of 1-DNJ Separation with 732 Resin}

The preparative separation of 1-DNJ was performed with 732 resin under optimal conditions. Through one treatment with the separation process, a yellowish powder was obtained with a recovery and purity of 1 -DNJ in the final product of $90.51 \%$ and $15.3 \%$. The results show that separation with 732 resin is a useful method in enriching 1-DNJ from mulberry leaves.

\section{Experimental}

\subsection{Materials}

The 1-DNJ standard was purchased from Tauto Biotech Co., Ltd (Shanghai, China). All other reagents were of analytical grade and deionized water was purified by a Milli-Q water-purification system from Millipore (Bedford, MA, USA). Mulberry leaves were collected from the royal ancient mulberry fields (Daxing District, Beijing, China) in July 2012 and identified by Professor Zhangji of National Institute for Food and Drug Control. The materials were cleaned and dried at $60{ }^{\circ} \mathrm{C}$, then powdered by a FW-100 herb disintegrator (Taisite Equipment Co., Ltd, Tianjin, China) and kept at $4{ }^{\circ} \mathrm{C}$.

Macroporous resins, including AB-8, 201, D101, D152, JK206, JK008, D113 and 732 were purchased from QiRui Petrochemical Company (Shandong, China). The adsorbent beads were pretreated to remove the monomersand porogenic agents trapped inside the pores during the synthesis process. Prior to use, the resins were soaked into ethanol (95\%), shaken for $24 \mathrm{~h}$ in room temperature and washed by deionized water thoroughly. The resins were placed into $\mathrm{HCl}(5 \%)$ and the same quantity of $\mathrm{NaOH}(5 \%)$ to remove impurities. Finally, the resins were washed by deionized water until the effluent was chemically neutral [24].

\subsection{The Extraction and Determination of 1-DNJ}

\subsubsection{The Extraction of 1-DNJ}

The mulberry powder was accurately weighed and refluxed under different extraction conditions (ethanol concentration, extraction temperature, extraction time and ratio of solvent to sample). After the 
extraction, the extracting solution was filtered and then concentrated under vacuum at $50{ }^{\circ} \mathrm{C}$. The residue was accurately weighted and subjected to HPLC system. The extraction yield was defined as the amount of 1-DNJ in the extracting solution from $100 \mathrm{~g}$ dry mulberry powder.

\subsubsection{Quantitative Determination of 1-DNJ}

The standard solutions of 1-DNJ or the extracted solutions were dissolved with appropriate amount of potassium borate buffer $(0.4 \mathrm{M}, \mathrm{pH} 8.5)$, and then $40 \mu \mathrm{L}$ FMOC-Cl $(5 \mathrm{mmol} / \mathrm{L})$ in $\mathrm{CH}_{3} \mathrm{CN}$ was added. The reactant was mixed immediately and allowed to react at $25{ }^{\circ} \mathrm{C}$ for $20 \mathrm{~min}$ in a water bath. $10 \mu \mathrm{L}$ amino acid $(0.1 \mathrm{M})$ was added to terminate the reaction by quenching the remaining FMOC-Cl. The mixture was diluted with $1 \mathrm{~mL}$ of $0.1 \%(v / v)$ aqueous acetic acid to stabilize the DNJ-FMOC, and filtered by a $0.45 \mu \mathrm{m}$ nylon syringe filter [25,26]. A $10 \mu \mathrm{L}$ aliquot of the filtrate was injected into the HPLC system. HPLC analysis was carried out on a 2695 Alliance separation module (Waters, Milford, MA, USA) equipped with a Waters temperature control module and a Waters 2475 fluorescence detector (excitation $254 \mathrm{~nm}$, emission $322 \mathrm{~nm}$ ). A Kromasil $\mathrm{C}_{18}$ column $(4.6 \mathrm{~mm} \times 250 \mathrm{~mm}, 5 \mu \mathrm{m})$ was used for the analysis. The analyte was eluted with a mobile phase of acetonitrile and $0.1 \%$ acetic acid $(50: 50, v / v)$ with flow rate $1.0 \mathrm{~mL} / \mathrm{min}$ at $30^{\circ} \mathrm{C}$.

\subsubsection{Experimental Designs}

RSM was used to find out the optimal extraction conditions for 1-DNJ. The extraction experiments were carried out according to a Box-Behnken design (BBD) with four factors and three levels. The four independent variables selected were ethanol concentration, extraction temperature, extraction time and ratio of solvent to sample as shown in Table 4. For each factor, the experimental range was based on the results of preliminary single-factor experiments. The yield of 1-DNJ extracted from mulberry leaves was the dependent variable. A total of 29 experiments were conducted to optimize the extraction conditions for the extraction procedures [27-30].

Table 4. Levels of the variables of Box-Behnken design (BBD).

\begin{tabular}{ccccc}
\hline Variables & \multirow{2}{*}{ Symbol } & \multicolumn{3}{c}{ Experimental Value } \\
\cline { 3 - 5 } & & Low, -1 & Zero, 0 & High, 1 \\
\hline Ethanol concentration $(\%)$ & $X_{1}$ & 50 & 60 & 70 \\
Extraction temperature $\left({ }^{\circ} \mathrm{C}\right)$ & $X_{2}$ & 70 & 80 & 90 \\
Extraction time $(\mathrm{h})$ & $X_{3}$ & 1 & 1.5 & 2 \\
ratio of solvent to sample $(\mathrm{mL} / \mathrm{g})$ & $X_{4}$ & 8 & 10 & 12 \\
\hline
\end{tabular}

\subsection{Static Adsorption and Desorption Tests}

The static adsorption tests of 1-DNJ with different resins were performed as follows: $5.0 \mathrm{~g}$ of hydrated test resins were placed in flasks and then $25 \mathrm{~mL}$ of sample solutions of mulberry leaves extracts (the concentration of 1-DNJ $6.95 \mathrm{mg} / \mathrm{mL}$ ) were added. The flasks were shaken (100 rpm) for $24 \mathrm{~h}$ at $25{ }^{\circ} \mathrm{C}$. The solutions after adsorption were analyzed by chromatography. The desorption processes were performed as follows: after reaching adsorption equilibrium, the resins were first 
washed by deionized water and then desorbed with $20 \mathrm{~mL}$ Ammonia $(1.0 \mathrm{~mol} / \mathrm{mL})$. The flasks were shaken $(100 \mathrm{rpm})$ for $24 \mathrm{~h}$ at $25^{\circ} \mathrm{C}$, then the desorption solutions were analyzed by chromatography.

\subsection{Static Adsorption Equilibrium Isotherms Tests}

The tests for equilibrium adsorption isotherms [31-34] with 732 and AB-8 resins were conducted by mixing $30 \mathrm{~mL}$ sample solutions of mulberry leaves extracts (the concentration of 1-DNJ $6.95 \mathrm{mg} / \mathrm{mL}$ ) at different concentrations with $5 \mathrm{~g}$ (dry weight) resins, and then shaking for $24 \mathrm{~h}$ at the temperature of $20{ }^{\circ} \mathrm{C}, 30{ }^{\circ} \mathrm{C}$ and $40{ }^{\circ} \mathrm{C}$. The initial and equilibrium concentrations at different temperatures were determined by chromatography.

The Langmuir isotherm theory is based on the assumption of adsorption on a homogeneous surface. The equation can be described in the following form:

$$
q_{e}=q_{m} \frac{k_{l} c_{e}}{1+k_{l} c_{e}}
$$

where $q_{e}$ is the equilibrium amount of adsorbent $(\mu \mathrm{g} / \mathrm{g}), c_{e}$ is the equilibrium concentration. The Freundlich equation is also used to describe the adsorption process. It can be written in the form:

$$
\ln q_{e}=\ln k_{f}+\left(\frac{1}{n_{f}}\right) \ln c_{e}
$$

The Toth model is applied to adsorption on heterogenerous surfaces. This equation is written in the following form:

$$
q_{e}=\frac{k_{T} c_{e}}{\left[a_{T}+c_{e}^{T}\right]^{1 / t}}
$$

\subsection{Static Adsorption Kinetic Tests}

The tests for adsorption kinetic [35,36] with 732 resin were conducted by mixing $50 \mathrm{~mL}$ sample solutions of mulberry leaves extracts (the concentration of 1-DNJ $6.95 \mathrm{mg} / \mathrm{mL}$ ) with $5.0 \mathrm{~g}$ (dry weight) resins, then shaken $(100 \mathrm{rpm})$ for $10 \mathrm{~h}$ at a temperature of $25^{\circ} \mathrm{C}$. The solutions were analyzed by chromatography at certain time till equilibrium.

The experimental data of adsorption rate constant were fitted to the two-step adsorption kinetics model to describe the adsorption kinetic property of solutions with the resin:

$$
\begin{gathered}
q_{t}=q_{e}-A_{1} e^{-k_{1} t}-A_{2} e^{-k_{2} t} \\
d \frac{q_{t}}{d t}=k_{i}\left(q_{e}-q_{t}\right)+c_{i} \quad i=1,2
\end{gathered}
$$

where $A_{1}$ and $A_{2}$ are frequency factors; $k_{1}$ and $k_{2}$ are adsorption rate constants; $q_{t}$ is the adsorption quantity (mg/g) [37]. 


\subsection{Dynamic Adsorption and Desorption Tests}

The dynamic adsorption and desorption tests were performed on glass $(25 \times 500 \mathrm{~mm})$ columns with a known dosage of 732 resins. Sample solution flowed through the glass columns at the rate of $5 \mathrm{~mL} / \mathrm{min}$. The concentration of 1-DNJ in the effluent liquid was analyzed by chromatography at certain time. After reaching adsorption equilibrium, the adsorbed column was washed by deionized water firstly, and then eluted by ammonia $(1 \mathrm{~mol} / \mathrm{mL})$ at $1 \mathrm{~mL} / \mathrm{min}$. The concentration of $1-\mathrm{DNJ}$ in desorption solution was determined by chromatography.

\subsection{The Preparative Separation of 1-DNJ with 732 Resin under Optimal Conditions}

The preparative separation of 1-DNJ was performed with 732 resin under optimal conditions. Sample solution was flowed through the glass columns at the rate of $5 \mathrm{~mL} / \mathrm{min}$ with the concentration of $9.84 \mathrm{mg} / \mathrm{mL}$. After reaching adsorption equilibrium, the adsorbed column was washed with four column volumes of deionized water and four column volumes of $10 \%$ ethanol. Ammonia $(1 \mathrm{~mol} / \mathrm{L})$ was selected to elute at the rate of $1 \mathrm{~mL} / \mathrm{min}$ and the eluent was concentrated under vacuum. The concentrated solution was diluted to a certain volume with deionized water and then spray drying to give the finally product. The product was weighed and subjected to chromatography.

\section{Conclusions}

The extraction and separation method of 1-DNJ from mulberry leaves with resins was successfully demonstrated in this study. The maximal yield of 1-DNJ was obtained from mulberry leaves when they were extracted under conditions of $55 \%$ ethanol concentration, extraction temperature of $80{ }^{\circ} \mathrm{C}$, extraction time of $1.2 \mathrm{~h}$ and ratio of solvent to sample of 12:1. Among the eight resins that were investigated, 732 resin offers the best separation capacity for 1-DNJ from other components in mulberry leaves extracts. Process parameters, including concentration and flow rate of sample solution, concentration and flow rate of desorption solution at different temperatures were optimized for the most effective separation of 1-DNJ from mulberry leaves with 732 resin. The equilibrium experimental data of adsorption of 1-DNJ from mulberry leaves with 732 resin at different temperatures fitted to Langmuir isotherms. The adsorption kinetics process for 1-DNJ in mulberry leaves with 732 resin fitted to two-step adsorption kinetics model. Through one run treatment on the column packed with 732 resin under optimal conditions, the recovery and purity of 1-DNJ in the final product was $90.51 \%$ and $15.3 \%$.

\section{Acknowledgments}

This work was financially supported by the Key Project of Chinese Ministry of Education (No.211003), the Scientific Research Common Program of Beijing Municipal Commission of Education (No.KM201110017002) and the BIPT Breeding Project of Outstanding Young Teachers and Management Backbones (No.BIPT-BPOYTMB-2013). 


\section{Author Contributions}

Cui-Qing Li and Teng Wang participated in designing the study. Teng Wang, Hong Zhang and Ji-Wen Li conducted the study and collected the data. Teng Wang and Hong Zhang analyzed the data and wrote the manuscript.

\section{Conflicts of Interest}

The authors declare no conflict of interest.

\section{References}

1. Naowaboot, J.; Pannangpetch, P.; Kukongviriyapan, V.; Kongyingyoes, B.; Kukongviriyapan, U. Antihyperglycemic, antioxidant and antiglycationactivities of mulberry leaf extract in streptozotocin-induced chronic diabetic rats. Plant Foods Hum. Nutr. 2009, 64, 116-121.

2. Chon, S.U.; Kim, Y.M.; Park, Y.J.; Heo, B.G.; Park, Y.S.; Gorinstein, S. Antioxidant and antiproliferative effects of methanol extracts from raw and fermented parts of mulberry plant (Morus alba L.). Eur. Food Res. Technol. 2009, 230, 231-237.

3. Yoshida, Y.; Hiradate, S.; Tsukamoto, T.; Hatakeda, K.; Shirata, A. Antimicrobial activity of culture filtrate of Bacillus amyloliquefaciens RC-2 isolated from mulberry leaves. Am. Phytopath. Soc. 2001, 91, 181-187.

4. Kooij, R.; Branderhorst, H.M.; Bonte, S.; Wieclawska, S.; Martin, N.I.; Pieters, R.J. Glycosidase inhibition by novel guanidinium and urea iminosugar derivatives. Med. Chem. Commun. 2013, 4, 387-393.

5. Tsuduki, T.; Kikuchi, I.; Kimura, T.; Nakagawa, K.; Miyazawa, T. Intake of mulberry 1-deoxynojirimycin prevents diet-induced obesity through increases in adiponectin in mice. Food Chem. 2013, 139, 16-23.

6. Liu, J.W.; Chen, Y.; Xiong, F.L. Studies on separation and purification of total flavones from Herba with macroreticular resin. Chin. Pharm. J. 2006, 16, 1222-1224.

7. Keka, S.; Papita, D.S.; Siddhartha, D. Extraction of natural dye from petals of Flame of forest (Butea monosperma) flower: Process optimization using response surface methodology (RSM). Dyes Pigm. 2012, 94, 212-216.

8. Lee, W.C.; Yusof, S.; Hamid, N.S.A.; Baharin, B.S. Optimizing conditions for hot water extraction of banana juice using response surface methodology (RSM). J. Food Eng. 2006, 75, 473-479.

9. Ahmad, A.L.; Derek, C.J.C.; Zulkali, M.M.D. Optimization of thaumatin extraction by aqueous two-phase system (ATPS) using response surface methodology (RSM). Sep. Purif. Technol. 2008, 62, 702-708.

10. Baş, D.; Boyacı, İ.H. Modeling and optimization I: Usability of response surface methodology. J. Food Eng. 2007, 78, 836-845.

11. Lai, J.X.; Xin, C.; Zhao, Y.; Feng, B.; He, C.F.; Dong, Y.M.; Fang, Y.; Wei, S.M. Optimization of Ultrasonic Assisted Extraction of Antioxidants from Black Soybean (Glycine max var) Sprouts Using Response Surface Methodology. Molecules 2013, 18, 1101-1110. 
12. Wang, X.S.; Wu, Q.N.; Wu, Y.F.; Chen, G.Y.; Yue, W.; Liang, Q.L. Response Surface Optimized Ultrasonic-Assisted Extraction of Flavonoids from Sparganii Rhizoma and Evaluation of Their in Vitro Antioxidant Activities. Molecules 2012, 17, 6769-6783.

13. Zou, T.B.; Xia, E.Q.; He, T.P.; Huang, M.Y.; Jia, Q.; Li, H.W. Ultrasound-Assisted Extraction of Mangiferin from Mango (Mangifera indica L.) Leaves Using Response Surface Methodology. Molecules 2014, 19, 1411-1421.

14. Asano, N.; Yamashita, T.; Yasuda, K. Polyhydroxylated alkaloids isolated from mulberry trees (Morus alba L.) and silkworms (Bombyx mori L.). Agric. Food Chem. 2001, 49, 4208-4213.

15. Kimura, M.; Chen, F. Antihyperglycemic effects of N-containing sugars derived from mulberry leaves is streptozocin-in-duced diabetic mice. Wakan Iyakugaku Zasshi 1995, 12, 214-219.

16. Dong, K. The overview of macroporous adsorptive resins in the study of Chinese herbal medicine. J. Pharm. Pract. 2006, 1, 13-16.

17. Mirhosseini, H.; Tan, C.P.; Hamid, N.S.A.; Yusof, S.; Chern, B.H. Characterization of the influence of main emulsion components on the physicochemical properties of orange beverage emulsion using response surface methodology. Food Hydrocolloid. 2009, 23, 271-280.

18. Pericin, D.; Popovic, S. Modeling and optimization of proteolytic process: Application of response surface methodology (RSM). J. Biotechnol. 2010, 150, S304.

19. Liu, X.Y.; Wang, Z.Y.; Wang, L.; Elfalleh, W.; Zhang, H. Ultrasonic-assisted extraction of polysaccharides from Hohenbueheliaserotina by response surface methodology. Int. J. Biol. Macromol. 2011, 51, 1-8.

20. Prakash, J.M.; Manikandanb, S.K.; Thirugnanasambandhama, C.; Vigna, N.R. Box-Behnken design based statistical modeling for ultrasound-assisted extraction of corn silk polysaccharide. Carbohyd. Polym. 2003, 92, 604-611.

21. Zinatizadeh, A.A.L.; Mohamed, A.R.; Abdullah, A.Z.; Mashitah, M.D.; Isa, M.H.; Najafpour, G.D. Process modeling and analysis of palm oil mill effluent treatment in an up-flow anaerobic sludge fixed film bioreactor using response surface methodology (RSM). Water Res. 2006, 40, 3193-3208.

22. Fu, B.Q.; Liu, J.; Li, H.; Li, L.; Lee, F.S.C.; Wang, X.R. The application of macroporous resins in the separation of licorice flavonoids andglycyrrhizic acid. J. Chromatogr. A 2005, 1089, 18-24.

23. Nagaoka, H.; Imae, T. Analytical investigation of two-step adsorption kinetics on surfaces. J. Colloid Interface Sci. 2003, 264, 335-342.

24. Xu, R.; Cheng, N.; Huang, W.; Gao, H.; Deng, J.; Cao, W. Effects of the processing steps on parathion levels during honey production and parathion removal by macroporous adsorption resins. Food Control 2012, 23, 234-237.

25. Kimura, T.; Nakagawa, K.; Saito, Y.; Yamagishi, K.; Suzuki, M.; Yamaki, K.; Shinmoto, H.; Miyazawa, T. Simple and rapid determination of deoxynojirimycin in mulberry leaves. Biofactors 2004, 22, 341-345.

26. Kim, J.W.; Kim, S.U.; Lee, H.S.; Kim, I.; Ahn, M.Y.; Ryu, K.S. Determination of 1-deoxynojirimycin in Morus alba L. leaves by derivatization with 9-fluorenylmethyl chloroformate followed by reversed-phase high-performance liquid chromatography. J. Chromatogr. A 2003, 1002, 93-99.

27. Chaluntorn, V.; Kiyotaka, N.; Phumon, S. Optimization of 1-deoxynojirimycin extraction from mulberry leaves by using response surface methodology. Biosci. Biotechnol. Biochem. 2009, 73, 2684-2689. 
28. Nakagawa, K.; Ogawa, K.; Higuchi, O.; Kimura, T.; Miyazawa, T.; Hori, M. Determination of iminosugars in mulberry leaves and silkworms using hydrophilic interaction chromatography-tandem mass spectrometry. Anal. Biochem. 2010, 404, 217-222.

29. Giovanni, M. Response surface methodology and product optimization. Food Technol. 1983, 37, 41-45.

30. Chaluntorn, V.; Kiyotaka, N.; Phumon, S.; Development of high 1-deoxynojirimycin (DNJ) content mulberry tea and use of response surface methodology to optimize tea-making conditions for highest DNJ extraction. Food Sci. Technol. 2012, 45, 226-232.

31. Ren, Y.; Wei, $\mathrm{X}$. Adsorption character for removal for $\mathrm{Cu}(\mathrm{II})$ by magnetic $\mathrm{Cu}$ (II) ion imprinted composite adsorbent. J. Hazard. Mater. 2008, 15, 14-22.

32. Lin, S.H.; Juang, R.S. Heavy metal removal from water by sorption using surfactant-modified montmorillonite. J. Hazard. Mater. 2002, 92, 315-326.

33. Feng, C.W.; Tseng, R.L.; Juang, R.S. Initial behavior of intraparticle diffusion model used in the description of adsorption kinetics. Chem. Eng. J. 2009, 153, 1-8.

34. Araceli, R.; Juan, G.; Gabriel, O.; María, M. Adsorption of anionic and cationic dyes on activated carbon from aqueous solutions: Equilibrium and kinetics. J. Hazard. Mater. 2009, 172, 1311-1320.

35. Yuan, Y.L. Equilibrium, thermodynamics and breakthrough studies for adsorption of solanesol onto macroporous resins, Chem. Eng. Process. 2008, 47, 1420-1427.

36. Zhong, Li.; Li, X.B.; Xi, H.X.; Hua, B. Effects of ultrasound on adsorption equilibrium of phenol on polymeric adsorption resin. Chem. Eng. J. 2002, 86, 375-379.

37. Jun, W.; Fu, A.W.; Hui, Z.; Li, L.; Qiu, S.W. Isolation of flavonoids from mulberry (Morus alba L.) leaves with macroporous resins. Afric. J. Biotechnol. 2008, 13, 2147-2155.

Sample Availability: Samples of the crude extract are available from the authors.

(C) 2014 by the authors; licensee MDPI, Basel, Switzerland. This article is an open access article distributed under the terms and conditions of the Creative Commons Attribution license (http://creativecommons.org/licenses/by/3.0/). 